\title{
Advances in Industrial Control
}

\section{Series editors}

Michael J. Grimble, Glasgow, UK

Michael A. Johnson, Kidlington, UK 
More information about this series at http://www.springer.com/series/1412 
Yihui Wang · Bin Ning - Ton van den Boom Bart De Schutter

\section{Optimal Trajectory Planning and Train Scheduling for Urban Rail Transit Systems}

望 Springer 
Yihui Wang

State Key Laboratory of Rail Traffic Control and Safety

Beijing Jiaotong University

Beijing

China

Bin Ning

State Key Laboratory of Rail Traffic Control and Safety

Beijing Jiaotong University

Beijing

China
Ton van den Boom

Delft Center for Systems and Control

Delft University of Technology

Delft

The Netherlands

Bart De Schutter

Delft Center for Systems and Control

Delft University of Technology

Delft

The Netherlands

MATLAB $^{\circledR}$ is a registered trademark of The MathWorks, Inc., 3 Apple Hill Drive, Natick, MA 01760-2098, USA, http://www.mathworks.com.

ISSN 1430-9491

Advances in Industrial Control

ISBN 978-3-319-30888-3

DOI 10.1007/978-3-319-30889-0
ISSN 2193-1577 (electronic)

ISBN 978-3-319-30889-0 (eBook)

Library of Congress Control Number: 2016933235

(C) Springer International Publishing Switzerland 2016

This work is subject to copyright. All rights are reserved by the Publisher, whether the whole or part of the material is concerned, specifically the rights of translation, reprinting, reuse of illustrations, recitation, broadcasting, reproduction on microfilms or in any other physical way, and transmission or information storage and retrieval, electronic adaptation, computer software, or by similar or dissimilar methodology now known or hereafter developed.

The use of general descriptive names, registered names, trademarks, service marks, etc. in this publication does not imply, even in the absence of a specific statement, that such names are exempt from the relevant protective laws and regulations and therefore free for general use.

The publisher, the authors and the editors are safe to assume that the advice and information in this book are believed to be true and accurate at the date of publication. Neither the publisher nor the authors or the editors give a warranty, express or implied, with respect to the material contained herein or for any errors or omissions that may have been made.

Printed on acid-free paper

This Springer imprint is published by Springer Nature

The registered company is Springer International Publishing AG Switzerland 


\section{Series Editors' Foreword}

The series Advances in Industrial Control aims to report and encourage technology transfer in control engineering. The rapid development of control technology has an impact on all areas of the control discipline. New theory, new controllers, actuators, sensors, new industrial processes, computer methods, new applications, new philosophies..., new challenges. Much of this development work resides in industrial reports, feasibility study papers, and the reports of advanced collaborative projects. The series offers an opportunity for researchers to present an extended exposition of such new work in all aspects of industrial control for wider and rapid dissemination.

The transportation sector of industry is the focus of much investment in infrastructure, vehicles, and technology. It is a sector highly dependent on control engineering for its operation, safety, reliability, and reduced environmental pollution. The Advances in Industrial Control monograph series tries to follow developments in this field by seeking texts that show the interaction between control (theory and applications) and the latest developments in transportation technology. It is interesting to examine the trends in various branches of transportation technology and view how the recent contributions to the Advances in Industrial Control monograph series are reporting such developments.

\section{Aerospace Control Systems}

Aerospace systems cannot exist without control and consequently there are always new developments in this field. There are two trends in terrestrial aerospace control systems that find expression in the series. The first is new aerospace structures as exemplified by the monographs:

- Active Control of Flexible Structures by Alberto Cavallo, Giuseppe De Maria, Ciro Natale, Salvatore Pirozzi (ISBN 978-1-84996-280-3, 2010); and

- Modeling and Control for a Blended Wing Body Aircraft edited by Martin Kozek and Alexander Schirrer (ISBN 978-3-319-10791-2, 2015). 
The second trend is that of control for rotorcraft aerial vehicles and in particular, for autonomous rotorcraft vehicles. Recent entries into the series include:

- Unmanned Rotorcraft Systems by Guowei Cai, Ben M. Chen, Tong Heng Lee (ISBN 978-0-85729-634-4, 2011);

- Quad Rotorcraft Control by Luis R. García Carrillo, Alejandro E. Dzul López, Rogelio Lozano, and Claude Pégard (ISBN 978-1-4471-4398-7, 2013); and

- Smart Helicopter Rotors by Ranjan Ganguli, Dipali Thakkar and Sathyamangalam R. Viswamurthy (ISBN 978-3-319-24766-3, 2015).

\section{Marine Control Systems}

As one of the oldest forms of transportation, control systems in the marine field are mature. However, even these systems are not immune from new concepts in control theory as the following monographs demonstrate:

- Robust Control of Diesel Ship Propulsion by Nikolaos Xiros (ISBN 978-1-85233-543-4, 2002);

- Ship Motion Control by Tristan Perez (ISBN 978-1-85233-959-3, 2005); and

- Control of Ships and Underwater Vehicles by Khac Duc Do and Jie Pan (ISBN 978-1-84882-729-5, 2009).

\section{Traffic and Automobile Control Systems}

For some years now the American Control Conference has fielded special sessions on automobile control systems. These sessions reflect the technological change caused by having new sensors, computers, and concomitant software as integral components of the automobile system. The series recently fielded three monographs in this growing "smart" car technology:

- Dry Clutch Control for Automotive Applications by Pietro J. Dolcini, Carlos Canudas de Wit and Hubert Béchart (ISBN 978-1-84996-067-0, 2010);

- Active Braking Control Systems Design for Vehicles by Sergio M. Savaresi and Mara Tanelli (ISBN 978-1-84996-349-7, 2010); and

- Optimal Control of Hybrid Vehicles by Bram de Jager, Thijs van Keulen and John Kessels (ISBN 978-1-4471-5075-6, 2015).

The ultimate outcome of "smart" car control technology must surely be the driverless car.

However, the proliferation of petrol- and diesel-fuelled automobiles and the rise of the long-distance commuter have created the problem of very severe air pollution in many global conurbations, for example in, Beijing, Delhi, Paris, Taipei, and London. Short-term solutions have involved the use of traffic-management schemes 
to reduce the number of vehicles entering these areas. The series was fortunate to publish a seminal monograph in this traffic-management field in 1998, namely:

- Feedback Control Theory for Dynamic Traffic Assignment by Pushkin Kachroo and Kaan M.A. Özbay (ISBN 978-1-4471-1209-9, 1998).

\section{Bus Urban Transit Systems}

The long-term solution being pursued in many global locations involves investment, and the construction and optimization of modern, safe, and reliable urban transit systems. These systems can involve a number of different transport modalities such as trams, buses, and overground and underground Metro systems. There is potential for control engineers to play a significant role in the development of such systems. An important monograph in the series that used recent control theory to analyze urban bus transportation was Hybrid Predictive Control for Dynamic Transport Problems by Alfredo A. Núñez, Doris A. Sáez and Cristián E. Cortés (ISBN 978-1-4471-4350-5, 2012).

\section{Rail and Rail-Based Urban Transit Systems}

Long-distance rail travel and rail-based urban transit systems are recognized as key solutions to moving large numbers of people from place to place with high levels of safety and reliability, and with minimized environmental pollution. Many new developments and upgrades of old systems are active worldwide. In 1995, the series was fortunate to publish the seminal monograph for rail control systems, Energy-Efficient Train Control by Philip G. Howlett and Peter J. Pudney (ISBN 978-1-4471-3086-4, 1995). Currently, these authors are involved with Amie R. Albrecht in a second edition of this monograph. In this new version the authors also recognize the importance of treating rail-based urban transit systems.

The survey ends with this new monograph addition to the series: Optimal Trajectory Planning and Train Scheduling for Urban Rail Transit Systems by Yihui Wang, Bin Ning, Ton van den Boom, and Bart De Schutter. This monograph is sharply focused on the optimization problems of urban rail transit systems and will be of interest to a wide range of readers from the control and transportation communities. It describes the challenge that exists for control engineers and, we hope it leads the way to more contributions in this important technological area. It is an excellent entry into the Advances in Industrial Control monograph series.

Industrial Control Centre

Glasgow

Scotland, UK
M.J. Grimble

M.A. Johnson 


\section{Preface}

Energy efficiency in transportation systems is becoming more and more important because of environmental concerns and for reasons of cost. For the Beijing urban rail transit system, the power consumption in 2008 was 6.5 million $\mathrm{kWh}$, which was $1 \%$ of the total power consumption of the city. It is predicted that the power consumption of Beijing urban rail transits system will rise to 13.9 million $\mathrm{kWh}$ in 2015, which would then account for $1.2 \%$ of the total power consumption. Furthermore, the energy consumption for the operation of trains is about $40-50 \%$ of the total power consumption for urban rail transit systems. Therefore, the reduction in energy consumption for the operation of trains is one of the key objectives in the operation of railway systems. Meanwhile, the interest of railway operators in energy efficiency has been increasing more and more in recent years, as even a small improvement in the energy consumption can make railway operators save a lot of money.

Furthermore, with the increase in passenger demand in large cities like Beijing, Shanghai, Tokyo, New York, and Paris, urban rail transit systems play an increasing role in the efficiency and sustainability of the overall transportation system. Nowadays the operation of trains in urban rail transit systems is characterized by a high frequency, where the minimum headway between two successive trains is usually $2-5 \mathrm{~min}$, which could be reduced to even $90 \mathrm{~s}$ with the development of advanced train control systems. When trains are operated at such a high frequency, their scheduling based on the passenger demand becomes more and more important for passenger satisfaction and for the reduction of operation costs. Passenger satisfaction depends on the waiting times, in-vehicle times, and the number of transfers, while the operation costs are determined by the number of train services and the energy consumption of train operations.

The aim of the book is to determine and develop mathematical models and solution approaches to shorten the travel time of passengers and to reduce energy consumption in urban rail transit systems. The content of Chaps. 1-7 can be summarized as follows: 
Chapter 1: A brief introduction to railway operations and the structure of this book are presented.

Chapter 2: The background material and literature review on the operation of trains and on urban rail train scheduling are presented.

Chapter 3: The optimal trajectory planning problem for the operation of a single train is considered. The varying line resistance, variable speed restrictions, and varying maximum traction force are included in the problem definition. The objective function is a trade-off between the energy consumption and the riding comfort. Two approaches are proposed to solve this optimal control problem:

- A pseudospectral method, i.e., a state-of-the-art method for optimal control, is applied for the first time in optimal train control, where the optimal trajectory planning problem is recast into a multiple-phase optimal control problem.

- The optimal trajectory planning problem is reformulated as a mixed integer linear programming (MILP) problem by approximating the nonlinear terms in the problem by piecewise affine functions. The resulting MILP problem can be solved efficiently by existing commercial and open source solvers.

The main conclusion of the chapter is that if the available time for computing the solution is large enough, the pseudospectral method is recommended since it has a higher performance. Otherwise, the MILP approach can be applied to obtain the optimal train trajectory.

Chapter 4: We investigate the optimal trajectory planning problem for multiple trains under fixed block signaling systems and moving block signaling systems. The constraints caused by the leading train in a fixed or moving block signaling system are included into the model for the operation of trains. Two solution approaches are proposed to solve the resulting optimal control problem for multiple trains:

- The greedy approach optimizes the trajectory of the leading train first and then based on the optimal trajectory of the leading train, the trajectory planning problem for the following train is solved. The MILP approach and the pseudospectral method are employed to solve the optimal trajectory for the leading train and the following train.

- The simultaneous approach optimizes the trajectory of all the trains in the problem formulation at the same time, where the MILP approach and the pseudospectral method are also applied.

In the simulation experiment, we show that the simultaneous MILP/pseudospectral approach yields a better performance but requires a higher computation time compared to the greedy MILP/pseudospectral approach.

Chapter 5: The real-time train scheduling problem for an urban rail transit line is investigated with the aim of minimizing the total travel time of passengers and the energy consumption of the operation of trains. The train scheduling problem in this chapter is based on origin-destination-independent (OD-independent) passenger demands, which means that the passenger arrival rates at stations do not depend on the origins and destinations of passengers but only on the number of passengers that 
arrived. With consideration of OD-independent passenger demands, the optimal departure times, running times, and dwell times are obtained by solving the scheduling problem for the urban rail transit system. Several approaches are proposed to solve this problem:

- A new iterative convex programming (ICP) approach is proposed to solve the train scheduling problem.

- Nonlinear programming approaches (sequential quadratic programing (SQP) and pattern search in particular), a mixed integer nonlinear programming approach, and an MILP approach are also applied to solve the problem.

We find that the ICP approach provides the best trade-off between performance and computational complexity comparing with other alternative solution approaches.

Chapter 6: We consider the train scheduling taking constant origin-destinationdependent (OD-dependent) passenger demands into account for an urban rail transit line, where the origins and destinations of passengers are considered in the passenger arrival rates at stations. A stop-skipping strategy is adopted to reduce the passenger travel time and the energy consumption. The train scheduling problem results in a mixed integer nonlinear programming problem, where two solution approaches are proposed:

- A bi-level approach is considered to solve the train scheduling problem, where the higher level optimizes the binary variables and the lower level solves a nonlinear non-convex problem for each combination of binary variables.

- We also propose a limited bi-level approach that first applies a threshold method to obtain a good initial solution for the problem and then limits the search space of the variables to speed up the optimization process.

Experiment indicates that the bi-level approach has better control performance, but the limited bi-level approach can provide an acceptable solution with much less computation time.

Chapter 7: An event-driven model is proposed for the train scheduling of an urban rail transit network, where a time-varying OD-dependent passenger demand, splitting of passenger flows, and passenger transfer behavior at transfer stations are included. This event-driven model involves three types of events, i.e., departure events, arrival events, and passenger arrival rate change events (which are used to describe the changes of passenger arrival rates at platforms due to time-varying passenger demand, passenger transfers, etc.) at platforms. The train scheduling problem that is constructed based on the event-driven model is a real-valued nonlinear non-convex programming problem. Several solution approaches, such as sequential quadratic programming (SQP), pattern search method, mixed integer linear programming, and genetic algorithms, can be applied to this train scheduling problem.

The simulation results of the case study show that the SQP method provides a better trade-off between control performance and computational complexity than the genetic algorithm. 
The models and approaches proposed in this book can be applied in urban rail transit systems to improve the train scheduling and train control in practice. In addition, they can also be adapted and extended to control and to optimize other transportation systems, such as bus transit systems, multi-car elevator systems, and automated guided vehicles.

This work is supported by the State Key Laboratory of Rail Traffic Control and Safety, Beijing Jiaotong University and the Delft Center for Systems and Control, Delft University of Technology. The authors thank Prof. Tao Tang for his support in publishing this book.

Beijing (China)

Yihui Wang

December 2015

Bin Ning

Ton van den Boom

Bart De Schutter 


\section{Contents}

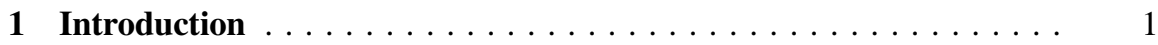

1.1 A Brief Introduction on Railway Operations . . . . . . . . 1

1.2 Book Outline ...................... 4

References ...................... 5

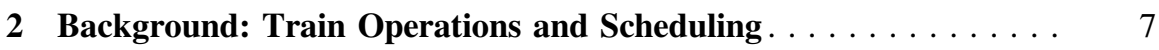

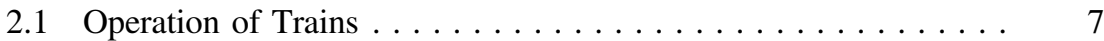

2.1.1 Automatic Train Operation . . . . . . . . . . . . . 7

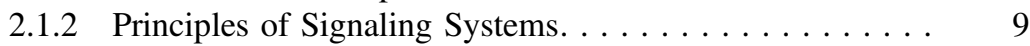

2.2 Optimal Trajectory Planning of Trains . . . . . . . . . . 11

2.2.1 Optimal Trajectory Planning of a Single Train . . . . . . 12

2.2.2 Optimal Trajectory Planning of Multiple Trains. . . . . . 13

2.3 Urban Rail Transit Scheduling Process . . . . . . . . . . . . . 14

2.3.1 Passenger Demand. . . . . . . . . . . . . . . . . . 15

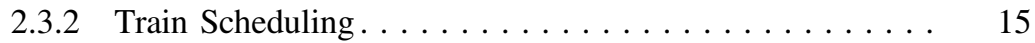

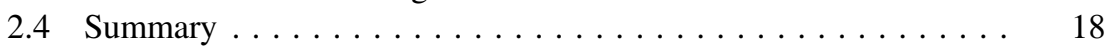

References ......................... 18

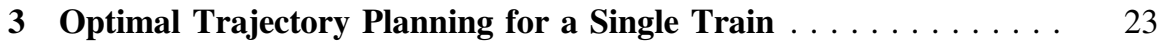

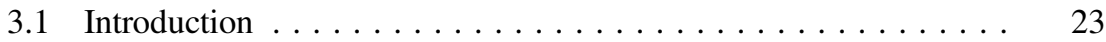

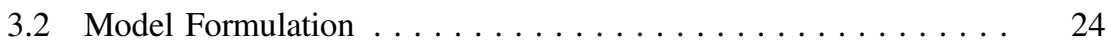

3.2 .1 Train Model . . . . . . . . . . . . . . . . . 24

3.2.2 An Assumption About the Line Resistance......... 26

3.3 Mathematical Formulation of the Single Train Trajectory

Planning Problem ....................... 27

3.4 Solution Approaches $\ldots \ldots \ldots \ldots \ldots \ldots \ldots \ldots \ldots \ldots$

3.4.1 Pseudospectral Method. . . . . . . . . . . . . . . . 30

3.4.2 Mixed Integer Linear Programming . . . . . . . . . . 33 


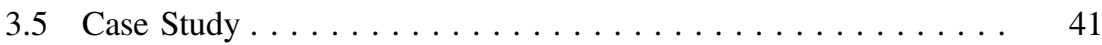

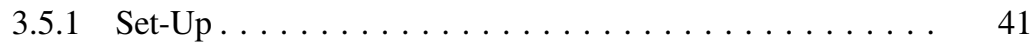

3.5.2 Results and Discussion. . . . . . . . . . . . . . 44

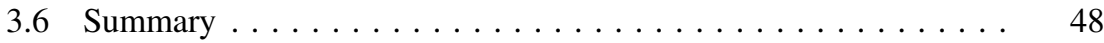

References ....................... 50

4 Optimal Trajectory Planning for Multiple Trains . . . . . . . . 53

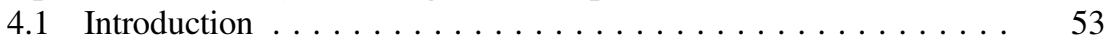

4.2 Model Formulation $\ldots \ldots \ldots \ldots \ldots \ldots \ldots \ldots \ldots \ldots \ldots$

4.2 .1 Train Dynamics. . . . . . . . . . . . . . . . 54

4.2.2 Operation of Trains in a Fixed Block Signaling System. . . . . . . . . . . . . . . . 55

4.2.3 Operation of Trains in a Moving Block Signaling System. . . . . . . . . . . . . . . . . 57

4.3 Mathematical Formulation of the Multiple Trains Trajectory

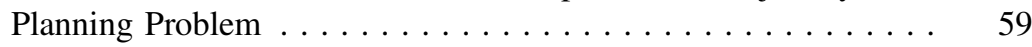

4.4 Solution Approaches $\ldots \ldots \ldots \ldots \ldots \ldots \ldots \ldots \ldots \ldots .6 \ldots$

4.4 .1 Greedy Approach $\ldots \ldots \ldots \ldots \ldots \ldots \ldots \ldots \ldots \ldots$

4.4.2 Simultaneous Approach . . . . . . . . . . . . . 61

4.5 Mixed Logical Dynamic Formulation for Signaling System Constraints . . . . . . . . . . . . . . . . 62

4.5.1 Multiple Trains Under Fixed Block Signaling System . . 62

4.5.2 Multiple Trains Under Moving Block Signaling System. . . . . . . . . . . . . . . . . . 65

4.5.3 Extension: Mode Vector Constraints . . . . . . . . . . 67

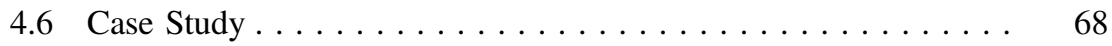

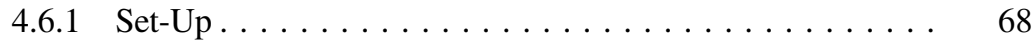

4.6.2 Results for the Fixed Block Signaling System. . . . . . . 71

4.6.3 Results for the Moving Block Signaling System . . . . . . 75

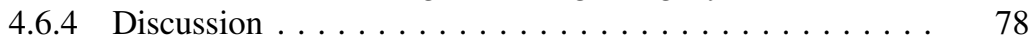

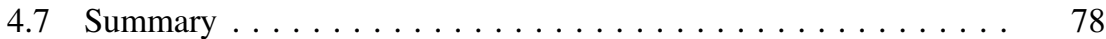

References ............................. 79

5 OD-Independent Train Scheduling for an Urban Rail

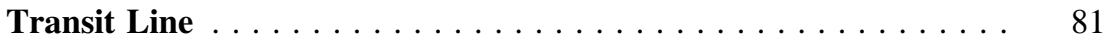

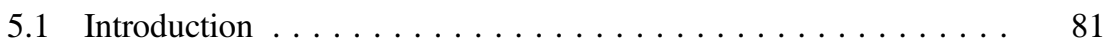

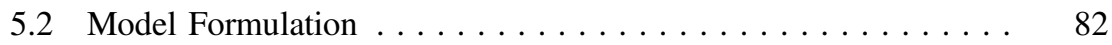

5.2.1 Arrivals and Departures ................ 83

5.2.2 Passenger Demand Characteristics . . . . . . . . . . 85

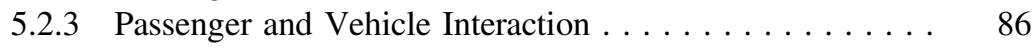

5.3 Mathematical Formulation of the Train Scheduling Problem .... 87

5.4 Solution Approaches . . . . . . . . . . . . . . . . . . 89

5.4.1 Gradient-Free Nonlinear Programming . . . . . . . . . . 89

5.4.2 Gradient-Based Nonlinear Programming . . . . . . . . . . 90

5.4.3 Mixed Integer Nonlinear Programming. . . . . . . . . . . . 90 
5.4.4 Mixed Integer Linear Programming . . . . . . . . . . . . 91

5.4.5 A New Approach: Iterative Convex Programming . . . . . . 92

5.5 Extension: Stop-Skipping at Small Stations . . . . . . . . . . . . 93

5.6 Case Study . . . . . . . . . . . . . . . . . . . . 95

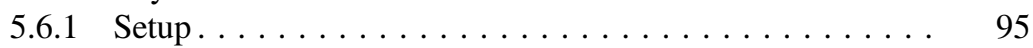

5.6.2 Results and Discussion. . . . . . . . . . . . . . . 99

5.7 Summary . . . . . . . . . . . . . . . . . . . . . . . . . 106

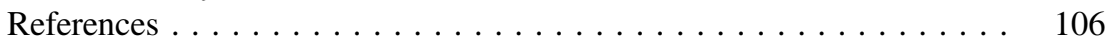

6 OD-Dependent Train Scheduling for an Urban Rail

Transit Line . . . . . . . . . . . . . . . . . . . . . . . . . . . . . . . . . . . 109

6.1 Introduction . . . . . . . . . . . . . . . . . . . . . . . . . . . . . . . . 109

6.2 Model Formulation . . . . . . . . . . . . . . . . . . . . . 110

6.2.1 Arrivals and Departures with Stop-Skipping . . . . . . . . . 112

6.2.2 OD-Dependent Passenger Demand Characteristics . . . . . . 114

6.3 Mathematical Formulation of the Scheduling Problem . . . . . . . 117

6.4 Solution Approaches . . . . . . . . . . . . . . . . . . . . . . . . 119

6.4.1 Bi-Level Optimization Approach . . . . . . . . . . . . . . . 119

6.4.2 Limited Bi-Level Optimization Approach . . . . . . . . . . 120

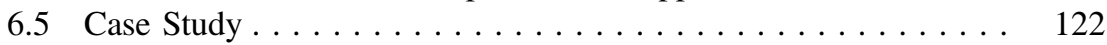

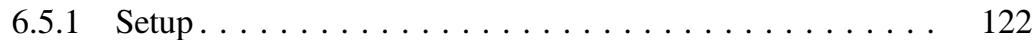

6.5 .2 Results and Discussion. . . . . . . . . . . . . . . . . . . . . . . . . . . . . . . . . 124

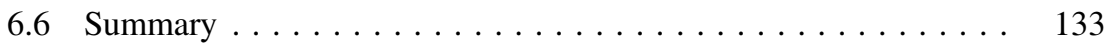

References .............................. 134

7 OD-Dependent Train Scheduling for an Urban Rail Transit

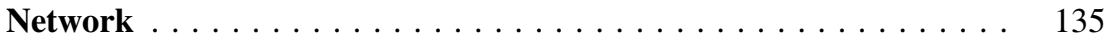

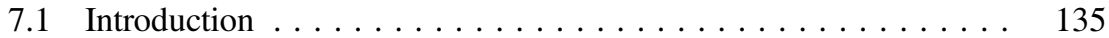

7.2 Model Formulation . . . . . . . . . . . . . . . . . 136

7.2.1 Three Types of Events . . . . . . . . . . . . . . 137

7.2 .2 Event-Driven Dynamics . . . . . . . . . . . . . . . . . . . . 142

7.3 Mathematical Formulation for the Scheduling Problem . . . . . . . . 147

7.3.1 Performance Criteria . . . . . . . . . . . . . . . . . . . . . . . . . . . . . . . . 147

7.3 .2 Constraints . . . . . . . . . . . . . . . . . . . . 148

7.4 Rolling Horizon Approach and Initial Conditions. . . . . . . . . . . 149

7.5 Solution Approaches . . . . . . . . . . . . . . . . . . . . . 150

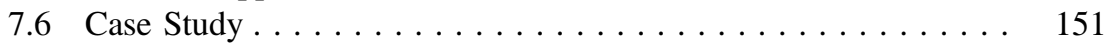

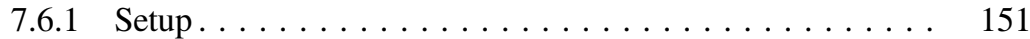

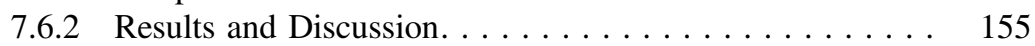

7.7 Summary . . . . . . . . . . . . . . . . . . . . . . . . . . . . . . . . . . . . . 159

References . . . . . . . . . . . . . . . . . . . . . . . . 159

8 Overview and Future Directions . . . . . . . . . . . . . . 161 
Appendix A: A General Formulation of the Pseudospectral

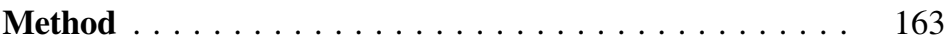

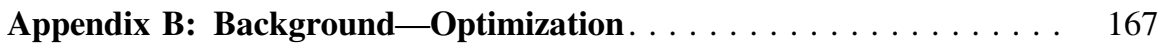

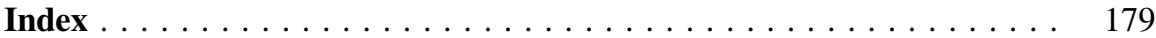




\section{Notation}

Below is a list of the most frequently used symbols and acronyms that occur in this book.

\section{Symbols}

\section{Chapter 3}

$\begin{array}{ll}m & \text { Mass }(\mathrm{kg}) \text { of a train } \\ g & \text { Gravitational acceleration }\left(\mathrm{m} / \mathrm{s}^{2}\right) \\ \rho & \text { Rotating mass factor }(-) \\ v & \text { Speed }(\mathrm{m} / \mathrm{s}) \text { of a train } \\ V_{\max }(s) & \text { Speed limit }(\mathrm{m} / \mathrm{s}) \text { along the track } \\ s & \text { Position }(\mathrm{m}) \text { of a train } \\ u & \text { Traction or braking force }(\mathrm{N}) \text { working on a train } \\ u_{\mathrm{max}}^{\text {acc }}, u_{\mathrm{max}}^{\mathrm{dec}} & \text { Maximum traction and braking force }(\mathrm{N}) \\ R_{b}(v) & \text { Roll resistance and air resistance }(\mathrm{N}) \text { of a train } \\ R_{\mathrm{l}}(s, v) & \text { Line resistance }(\mathrm{N}) \text { caused by track grade, curves, and tunnels } \\ \alpha(s) & \text { Slope along the track (rad) } \\ r(\mathrm{~s}) & \text { Radius }(\mathrm{m}) \text { of the curve along the track } \\ l_{\mathrm{t}}(\mathrm{s}) & \text { Length (m) of tunnels along the track } \\ f_{\mathrm{c}}(\mathrm{r}(\mathrm{s})) & \text { Curve resistance }(\mathrm{N}) \\ f_{\mathrm{t}} & \text { Tunnel resistance }(\mathrm{N}) \\ T & \text { Given running time }(\mathrm{s}) \text { for a train } \\ s_{k} & \text { Boundary (m) of discrete space interval } k \\ \Delta s_{k} & \text { Length }(\mathrm{m}) \text { of discrete space interval } k \\ E(k) & \text { Kinetic energy }(\mathrm{J}) \text { of a train at position } s_{k} \\ E_{\min } & \text { Minimum kinetic energy }(\mathrm{J}) \\ t(k) & \text { Passing time }(\mathrm{s}) \text { of a train at position } s_{k}\end{array}$




\section{Chapter 4}

$v_{\max }$

$v_{\text {yellow }}$

$v_{\min }$

$H_{\text {min,FBS }}$

$H_{\text {min MBS }}$

$s_{\mathrm{FB}, m}$

$L_{\mathrm{a}}$

$L_{\mathrm{t}}^{\mathrm{L}}$

$L_{\mathrm{S}}$

$S_{\mathrm{SM}}$

$v_{\text {max }}^{\mathrm{F}}$

$t_{\mathrm{r}}^{\mathrm{F}}$

$L_{\mathrm{r}}^{\mathrm{F}}$

$a_{\mathrm{dec}}^{\mathrm{F}}$

$t_{\mathrm{dec}}^{\mathrm{F}}$

$t_{\mathrm{d}}^{\mathrm{L}}$

$v^{\mathrm{F}}(t)$

$a_{\mathrm{acc}}^{\mathrm{L}}$

$t_{\text {safe }}^{\mathrm{F}}$

\section{Chapter 5}

$i$

j

$s_{j}$

$d_{i, j}$

$a_{i, j}$

$\tau_{i, j}$

$r_{i, j}$

$v_{i, j}$

$a_{i, j}^{\mathrm{acc}}$

$a_{i, j}^{\mathrm{dec}}$

$h_{0}$

$k_{1 i}, k_{2 i}, k_{3 i}$

$\lambda_{j}$

$\rho_{j}$
Speed code $(\mathrm{m} / \mathrm{s})$ for a green signal aspect in a three-aspect FBS system

Speed code $(\mathrm{m} / \mathrm{s})$ for a yellow signal aspect in a three-aspect FBS system

Speed code $(\mathrm{m} / \mathrm{s})$ for a red signal aspect in a three-aspect FBS system

Minimum headway (s) between trains in an FBS system

Minimum headway (s) between trains in an MBS system

Boundary (m) of fixed block section $m$

Length (m) of a block in an FBS system

Length $(m)$ of the leading train

Length $(\mathrm{m})$ of the secure section

Safety margin $(\mathrm{m})$ for braking of the following train

Maximum speed $(\mathrm{m} / \mathrm{s})$ of the following train

Reaction time (s) of the driver and equipment of the following train

Distance (m) that the following train may travel during the reaction time

Deceleration $\left(\mathrm{m} / \mathrm{s}^{2}\right)$ of the following train

Deceleration time (s) of the following train

Station dwell time (s) of the leading train

Speed $(\mathrm{m} / \mathrm{s})$ of the following train at time instant $t$

Acceleration $\left(\mathrm{m} / \mathrm{s}^{2}\right)$ of the leading train

Safety time margin (s) caused by safety distance margin and train length

\section{Train number}

Station number

Track section length $(\mathrm{m})$ between station $j$ and station $j+1$

Departure time (s) of train $i$ at station $j$

Arrival time (s) of train $i$ at station $j$

Dwell time (s) of train $i$ at station $j$

Running time (s) of train $i$ at station $j$

Train speed $(\mathrm{m} / \mathrm{s})$ of the holding phase for train $i$ on segment $j$

Acceleration $\left(\mathrm{m} / \mathrm{s}^{2}\right)$ for train $i$ on segment $j$

Deceleration $\left(\mathrm{m} / \mathrm{s}^{2}\right)$ for train $i$ on segment $j$

Minimum headway between two successive trains

Resistance coefficients for train $i$

Passenger arrival rate (passenger/s) at station $j$

Passenger alighting proportion when a train arrives at station $j$ 


$C_{i, \text { max }}$
$w_{i, j}^{\text {wait }}$
$w_{i, j}$
$n_{i, j}^{\text {remain }}$
$n_{i, j}^{\text {board }}$
$n_{i, j}^{\text {alight }}$
$n_{i, j}$
$\tau_{i, j \text { max }}, \tau_{i, j}$, min
$\alpha_{1, d}, \ldots, \alpha_{4, d}$
$n_{\text {door }}$
$t_{\text {wait }, i, j}$
$t_{\text {in-vehicle }, i, j}$
$E_{i, j}^{\text {acc }}$
$E_{i, j}^{\text {hold }}$
$E_{i, j}^{\text {dec }}$
$t_{\text {total,nom }}$
$E_{\text {total,nom }}$

Chapter 6

$S_{\text {skip }}$
$h_{0, \text { dep }}$
$h_{0, \text { arr }}$
$\tau_{0, \text { min }}$
$C_{0}^{\text {her }}$
$y_{i, j}$
$\lambda_{j, m}$
$w_{i, j, m}^{\text {wait }}$
$w_{i, j, m}$
$w_{i, j}^{\text {want-to-board }}$
$n_{i, j, m}^{\text {board }}$

Maximum capacity (passengers) of train $i$

Number of passengers waiting for train $i$ at station $j$

Number of passengers left immediately after the departure of train $i$ at station $j$

Remaining capacity (passengers) of train $i$ at station $j$ after the passenger alighting process

Number of passengers boarding train $i$ at station $j$

Number of passengers alighting from train $i$ at station $j$

Number of passengers on train $i$ when it departs from station $j$

Maximum and minimum dwell time (s)

Coefficients of the minimum dwell time

Number of doors of the train

Passenger waiting time (s) at station $j$ for train $i$

Passenger in-vehicle time (s) at station $j$ for train $i$

Energy consumption $(\mathrm{J})$ of the acceleration phase at station $j$ for train $i$

Energy consumption ( $\mathrm{J}$ ) of the holding phase at station $j$ for train $i$

Energy consumption $(\mathrm{J})$ of the deceleration phase at station $j$ for train $i$

Nominal value (s) of the travel time of passengers

Nominal value $(\mathrm{J})$ of the total energy consumption

Skipping set

Minimum departure headway (s) at terminal station

Minimum arrival headway (s) at terminal station

Minimum dwell time (s) at terminal station

Capacity (passengers) of the terminal station

Binary variable to indicate whether train $i$ stops at station $j$ or not

Passenger arrival rate (passengers/s) at station $j$ for passenger with destination $m$

Number of passengers with destination $m$ waiting for train $i$ at station $j$

Number of passengers with destination $m$ remaining at station $j$ immediately after the departure of train $i$

Number of passengers who want to board train $i$ at station $j$

Number of passengers with destination $m$ boarding train $i$ at station $j$ 
Chapter 7

$S_{\mathrm{ln}}$

$S_{\text {sta }}$

$S_{\text {pal }}$

$p$

$\mathfrak{p}^{\text {pla }}(p)$

$\mathfrak{s}^{\text {pla }}(p)$

$\mathfrak{p}^{\text {tra }}(i)$

$\mathfrak{s}^{\text {tra }}(i)$

$e_{k}$

$t_{k}$

$Y_{\text {type }, k}$

$i_{k}$

$p_{k}$

$\lambda_{j, m}^{\text {station }}$

$\beta_{p, m}^{\text {station }}$

$a_{0, p}^{\text {walk }}, b_{0, p}^{\text {walk }}$

$\theta_{p}^{\text {walk-in }}$

$t_{p}^{\mathrm{walk}-\mathrm{in}}$

$\beta_{i, p, m}^{\text {train }}$

$t_{p}^{\text {walk-out }}$

$a_{p, p^{\prime}}^{\mathrm{walk}}, b_{p, p^{\prime}}^{\mathrm{walk}}$

$\theta_{i, p, p^{\prime}}^{\text {walk }}$

$a_{p, p^{\prime}}^{\text {duration }}, b_{p, p^{\prime}}^{\text {duration }}$

$\theta_{i, p, p^{\prime}}^{\text {duration }}$

$w_{p_{k}}^{\text {wait,before }}$

$w_{p_{k}}^{\text {wait,after }}$
Set of urban rail transit lines

Set of stations

Set of platforms

Platform index

Predecessor of platform $p$

Successor of platform $p$

Predecessor of train $i$

Successor of train $i$

Event $k$

Time instant (s) at which event $e_{k}$ occurs

Event type (departure, arrival, $\lambda$-change) of event $k$

Index of the train corresponding to event $e_{k}$

Index of the platform corresponding to event $e_{k}$

Passenger arrival rates (passengers/s) at station $j$ with destination $m$

Splitting rate of passenger flows that arrive at station $j$ with destination $m$ and that go to platform $p$

Coefficients for average walking time from the entrance to platform $p$

Average walking time (s) for passengers from entrances to platform $p$

Total walking time (s) for passengers from entrances to platform $p$

Splitting rate to platform $p$ for passengers that are on board of train $i$ and have destination $m$

Total walking time (s) for passengers from platform $p$ to exit station

Coefficients for average walking time from platform $p$ to platform $p^{\prime}$

Average walking time (s) for transfer passengers alighting from train $i$ from platform $p$ to platform $p^{\prime}$

Coefficients for the duration time of the transfer process from platform $p$ to platform $p^{\prime}$

Duration time (s) for the transfer process from platform $p$ to platform $p^{\prime}$ for train $i$

Number of passengers waiting at platform $p_{k}$ before event $e_{k}$ occurs

Number of passengers waiting at platform $p_{k}$ immediately after event $e_{k}$ 


\section{Abbreviations}

The following abbreviations are used in this book:

ATO

ATP

ATS

DP

FBS

ICP

MBS

MILP

MINLP

PWA

SQP

OD-dependent

OD-independent
Automatic train operation

Automatic train protection

Automatic train supervision

Dynamic programming

Fixed blocking signaling

Iterative convex programming

Moving blocking signaling

Mixed integer linear programming

Mixed integer nonlinear programming

Piecewise-affine

Sequential quadratic programming

Origin-destination-dependent

Origin-destination-independent 